\title{
The role of steroids in the management of brain metastases: a systematic review and evidence-based clinical practice guideline
}

\author{
Timothy C. Ryken • Michael McDermott $\cdot$ Paula D. Robinson • \\ Mario Ammirati - David W. Andrews • Anthony L. Asher · Stuart H. Burri • \\ Charles S. Cobbs • Laurie E. Gaspar • Douglas Kondziolka • Mark E. Linskey • \\ Jay S. Loeffler • Minesh P. Mehta $\cdot$ Tom Mikkelsen · Jeffrey J. Olson • \\ Nina A. Paleologos $\cdot$ Roy A. Patchell $\cdot$ Steven N. Kalkanis
}

Received: 8 September 2009/Accepted: 8 November 2009/Published online: 3 December 2009

(C) The Author(s) 2009. This article is published with open access at Springerlink.com

\begin{abstract}
Question

Do steroids improve neurologic symptoms in patients with metastatic brain tumors compared to no treatment? If steroids are given, what dose should be used? Comparisons include: (1) steroid therapy versus none. (2) comparison of different doses of steroid therapy.

\section{Target population}

These recommendations apply to adults diagnosed with brain metastases.

\section{Recommendations}

\section{Steroid therapy versus no steroid therapy}

Asymptomatic brain metastases patients without mass effect Insufficient evidence exists to make a treatment recommendation for this clinical scenario.

Brain metastases patients with mild symptoms related to mass effect

Level 3 Corticosteroids are recommended to provide temporary symptomatic relief of symptoms related to increased intracranial pressure and edema secondary to brain metastases. It is recommended for patients who are symptomatic from metastatic disease to the brain that a starting dose of 4 $8 \mathrm{mg} /$ day of dexamethasone be considered.
\end{abstract}

T. C. Ryken

Department of Neurosurgery, Iowa Spine and Brain Institute,

Iowa City, IA, USA

M. McDermott

Department of Neurosurgery, University of California

San Francisco, San Francisco, CA, USA

P. D. Robinson

McMaster University Evidence-Based Practice Centre,

Hamilton, ON, Canada
Brain metastases patients with moderate to severe symptoms related to mass effect

Level 3 Corticosteroids are recommended to provide temporary symptomatic relief of symptoms related to increased intracranial pressure and edema secondary to brain metastases. If patients exhibit severe symptoms consistent with increased intracranial pressure, it is recommended that higher doses such as $16 \mathrm{mg} /$ day or more be considered.

\section{Choice of Steroid}

Level 3 If corticosteroids are given, dexamethasone is the best drug choice given the available evidence.

Duration of Corticosteroid Administration

Level 3 Corticosteroids, if given, should be tapered slowly over a 2 week time period, or longer in symptomatic patients, based upon an individualized treatment regimen and a full understanding of the long-term sequelae of corticosteroid therapy.

Given the very limited number of studies (two) which met the eligibility criteria for the systematic review, these are the only recommendations that can be offered based on this methodology. Please see "Discussion" and "Summary" section for additional details.

\footnotetext{
M. Ammirati

Department of Neurosurgery, Ohio State University

Medical Center, Columbus, OH, USA

D. W. Andrews

Department of Neurosurgery, Thomas Jefferson

University, Philadelphia, PA, USA

A. L. Asher

Department of Neurosurgery, Carolina Neurosurgery and Spine Associates, Charlotte, NC, USA
} 
Keywords Brain metastases - Steroids . Neurologic symptoms $\cdot$ Steroid dosage . Systematic review $\cdot$ Practice guideline

\section{Rationale}

Glucocorticoids have typically been used to assist in controlling cerebral edema in the early supportive care of the patient with newly diagnosed metastatic brain disease. Dexamethasone is generally considered the steroid of choice because of its minimal mineralocorticoid effect and long half-life, although any other corticosteroid can be effective if given in equipotent doses. Steroids have been used alone for palliation of symptoms and in combination with radiotherapy as an initial course of therapy. A review of the available literature indicates that the majority of these patients have been managed with starting doses of 4$8 \mathrm{mg} /$ day and it has been stated that up to $75 \%$ of patients with brain metastases show marked neurological improvement within 24-72 h after beginning dexamethasone [1]. However, side effects from chronic dexamethasone administration, including myopathy, are frequent and contribute to disability. Asymptomatic or minimally symptomatic patients may benefit little from the routine administration of steroid therapy and be exposed to these toxicities. Although successful clinical experience would lead to the conclusion that the role of steroids is firmly established in the management of brain metastases, even a

\section{S. H. Burri}

Department of Radiation Oncology,

Carolinas Medical Center, Charlotte, NC, USA

C. S. Cobbs

Department of Neurosciences, California Pacific Medical Center, San Francisco, CA, USA

\section{E. Gaspar}

Department of Radiation Oncology, University

of Colorado-Denver, Denver, CO, USA

D. Kondziolka

Department of Neurological Surgery, University

of Pittsburgh Medical Center, Pittsburgh, PA, USA

\section{E. Linskey}

Department of Neurosurgery, University of California-Irvine Medical Center, Orange, CA, USA

\section{J. S. Loeffler}

Department of Radiation Oncology, Massachusetts General

Hospital, Boston, MA, USA

\section{P. Mehta}

Department of Human Oncology, University of Wisconsin

School of Public Health and Medicine, Madison, WI, USA cursory review of the literature will demonstrate significant variability in recommendations and a general lack of wellcontrolled studies addressing this specific issue.

This systematic review addresses the role of corticosteroids in the treatment of metastatic brain disease with the following overall objectives:

1. To systematically review the evidence available for the following treatment comparisons for patients diagnosed with brain metastases specifically addressing the following questions:

- Do steroids improve neurologic symptoms in patients with metastatic brain tumors compared to no treatment?

- If steroids are given, what dose should be used?

2. To make recommendations based on this evidence for the role of corticosteroids in the management of these patients.

\section{Methods}

\section{Search strategy}

The following electronic databases were searched from 1990 to September 2008: MEDLINE ${ }^{\circledR}$, Embase $^{\circledR}$, Cochrane Database of Systematic Reviews, Cochrane Controlled Trials Registry, and Cochrane Database of Abstracts

T. Mikkelsen

Department of Neurology, Henry Ford Health System, Detroit, MI, USA

J. J. Olson

Department of Neurosurgery, Emory University

School of Medicine, Atlanta, GA, USA

N. A. Paleologos

Department of Neurology, Northshore University

Health System, Evanston, IL, USA

R. A. Patchell

Department of Neurology, Barrow Neurological Institute, Phoenix, AZ, USA

S. N. Kalkanis $(\square)$

Department of Neurosurgery, Hermelin Brain Tumor Center, Henry Ford Health System, 2799 West Grand Blvd, K-11,

Detroit, MI 48202, USA

e-mail: kalkanis@neuro.hfh.edu; skalkan1@hfhs.org 
of Reviews of Effects. A broad search strategy using a combination of subheadings and text words was employed. The search strategy is documented in the methodology paper for this guideline series by Robinson et al. [2]. Reference lists of included studies were also reviewed.

\section{Eligibility criteria}

- Published in English with a publication date of 1990 forward.

- Patients with brain metastases.

- Fully-published peer-reviewed primary comparative studies (all comparative study designs for primary data collection included; e.g., RCT, non-randomized trials, cohort studies or case-control studies).

- Study comparisons include one or more of the following:

- steroid therapy versus none.

- comparison of different doses of steroid therapy.

- Number of study participants with brain metastases $\geq 5$ per study arm for at least two of the study arms.

- Baseline information on study participants is provided by treatment group in studies evaluating interventions exclusively in patients with brain metastases. For studies with mixed populations (i.e., includes participants with conditions other than brain metastases), baseline information is provided for the intervention sub-groups of participants with brain metastases.

Study selection and quality assessment

Two independent reviewers evaluated citations using a priori criteria for relevance and documented decisions in standardized forms. Cases of disagreement were resolved by a third reviewer. The same methodology was used for full text screening of potentially relevant papers. Studies which met the eligibility criteria were data extracted by one reviewer and the extracted information was checked by a second reviewer. The PEDro scale was used to rate the quality of randomized trials [3, 4]. The quality of comparative studies using non-randomized designs was evaluated using eight items selected and modified from existing scales as outlined in Appendix B of the Methodology chapter in this brain metastases guideline series [2].

Evidence classification and recommendation levels

Both the quality of the evidence and the strength of the recommendations were graded according to the criteria endorsed by the American Association of Neurological Surgeons (AANS) and the Congress of Neurological
Surgeons (CNS). These criteria are provided in the methodology paper of this guideline series.

Guideline development process

The AANS/CNS convened a multi-disciplinary panel of clinical experts to develop a series of practice guidelines on the management of brain metastases based on a systematic review of the literature conducted in collaboration with methodologists at the McMaster University Evidencebased Practice Center.

\section{Scientific foundation}

Despite the widespread use of steroids in the management of brain metastases, only two publications met the stated eligibility criteria [5, 6]. Figure 1 outlines the flow of studies through the review process. These two studies are outlined in the attached evidentiary table. In order to expand the information base for the "Discussion" section of this chapter, additional searches were undertaken by reviewing the bibliographies of these two papers and additional review of the published literature addressing the treatment of metastatic brain disease for references to steroid administration. These articles are summarized in the "Discussion".

Studies meeting search criteria

The only two papers that met the search criteria are summarized below $[5,6]$. The details are also summarized in the accompanying evidentiary table (Table 1). In expanding the literature review to seek out additional supporting information for the "Discussion" portion of this document, we did not encounter any additional high quality studies specifically addressing the role of steroids in brain

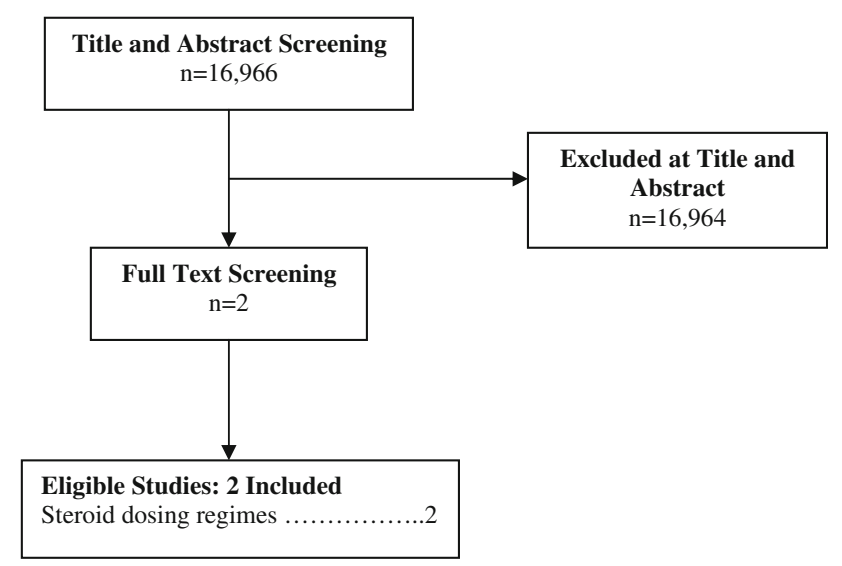

Fig. 1 Flow of studies to final number of eligible studies 


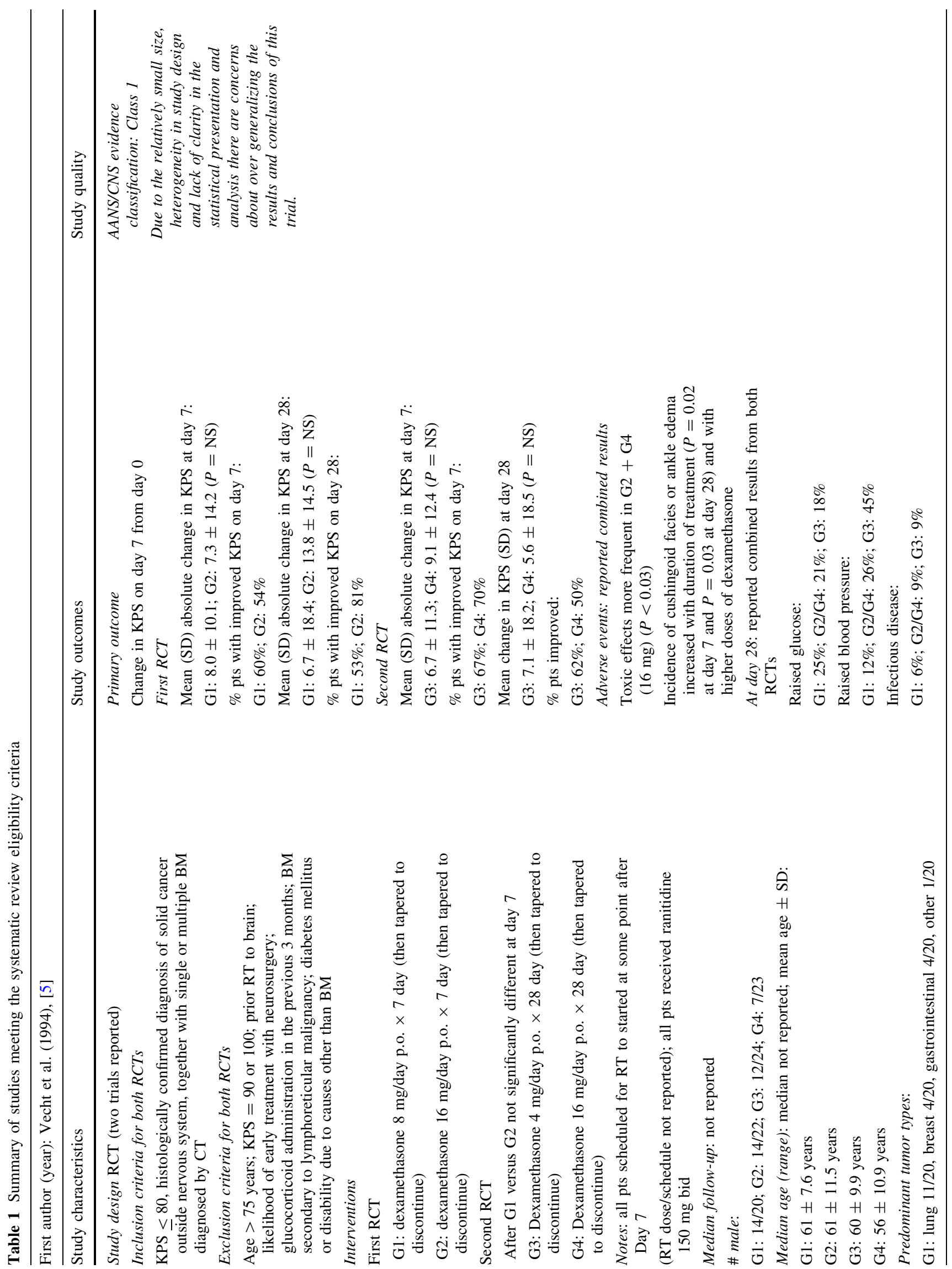




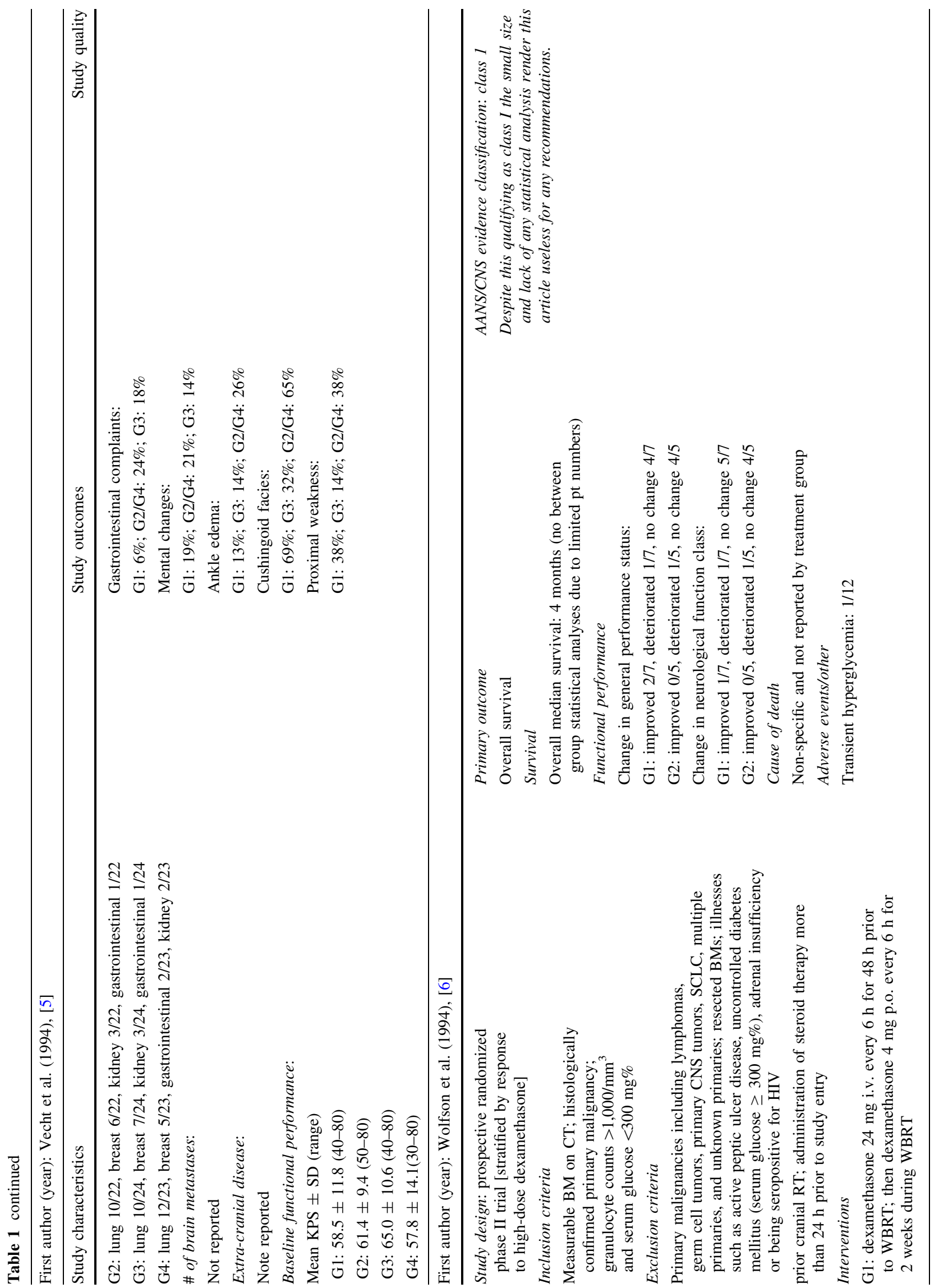




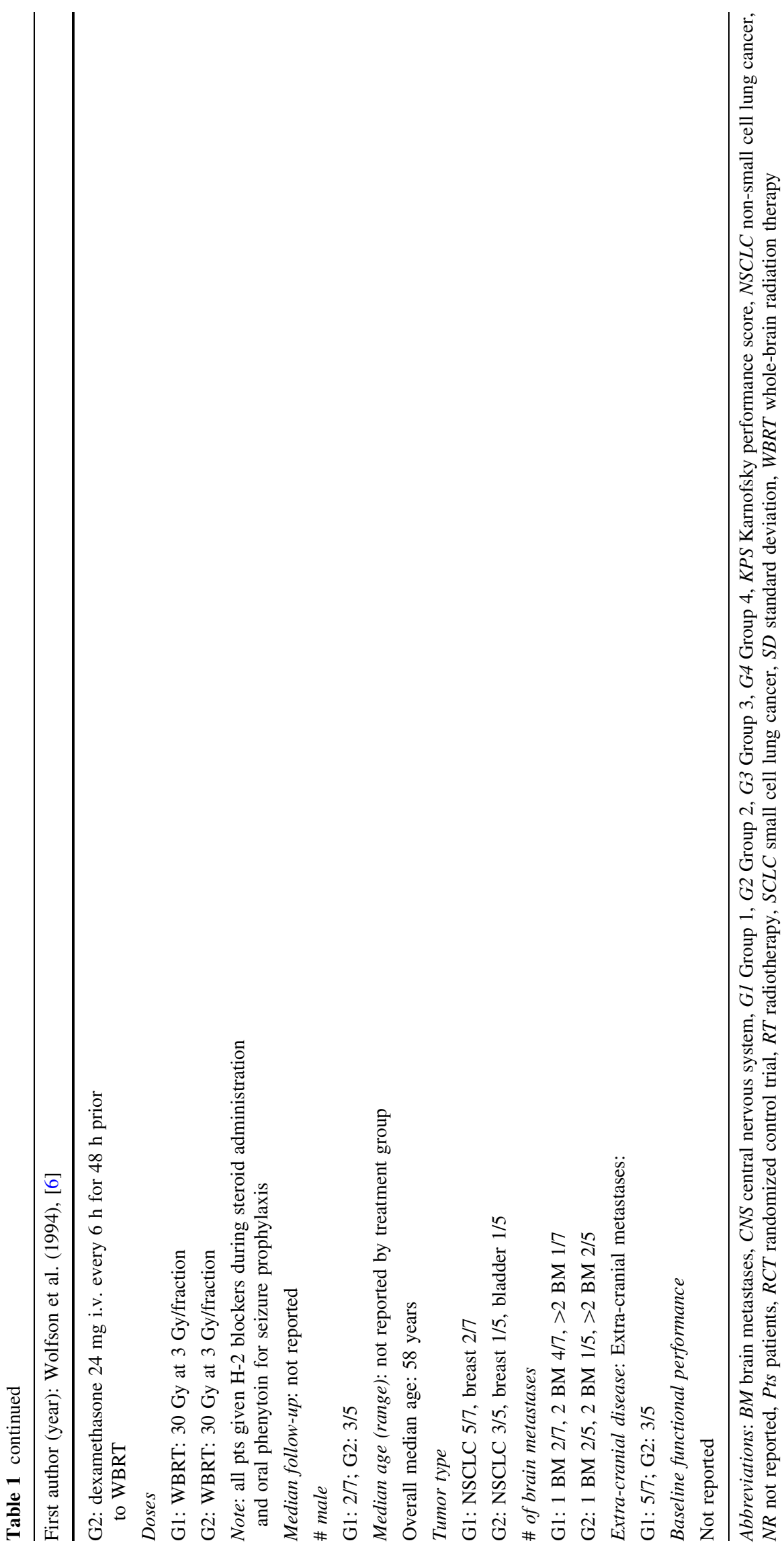


metastases. This was in agreement with several previous authors who upon review of the topic have encountered a lack of detailed, controlled accounts of steroid use in the studies under review, including the large randomized controlled trials that often form the basis of treatment decisions and recommendations [1, 7-9].

The first of the two studies to meet the search criteria described a randomized study of 4,8 and $16 \mathrm{mg} /$ day dosing of dexamethasone and found no advantage to higher dosing in patients who were not felt to be in impending danger of cerebral herniation [5]. Vecht et al., published their findings from two consecutively executed double-blind randomized trials in patients with brain metastases and Karnofsky performance scores (KPS) of 80 or less which were designed to evaluate the minimum effective dose of oral dexamethasone. Initially a dexamethasone dosage of $8 \mathrm{mg} /$ day (Group 1) was compared to $16 \mathrm{mg} /$ day (Group 2), followed by a comparison of $4 \mathrm{mg} /$ day (Group 3) versus $16 \mathrm{mg} /$ day (Group 4). The outcomes of interest were alteration in KPS and the frequency of side effects at days $0,7,28$, and 56 .

In the initial study, although both groups showed an improvement, there was no significant difference in KPS improvement comparing the 8-mg group versus the 16-mg group at day 7 (mean $8.0 \pm 10.1$ versus $7.3 \pm 14.2$ ). At the 28 day point, there was a larger but still non-significant rate of improvement thought to be biased by earlier steroid tapering in the lower group.

In the second trial, again both groups showed improvement. However, no significant difference between the 4- and 16-mg group (comparing $6.7 \pm 11.3$ points at day 7 and $7.1 \pm 18.2$ points at day 28 , versus $9.1 \pm 12.4$ and $5.6 \pm 18.5$ points, respectively) could be detected. Side effects (Cushingoid changes and extremity edema) occurred more frequently in the $16 \mathrm{mg} / \mathrm{day}$ versus the $4 \mathrm{mg} / \mathrm{day}$ group at Day 28 (Combined frequency $91 \%$ versus $46 \%, P<0.03$ ).

The authors conclude that for the majority of patients, the lower doses of 4 and $8 \mathrm{mg}$ dexamethasone per day have an equivalent effect on improving neurologic performance when compared to a dose of $16 \mathrm{mg} /$ day at 1 and 4 weeks of treatment, in moderately symptomatic patients without signs of impending herniation. A non-significant trend toward improvement at 28 days was noted with the higher dosage. The authors postulated that the early taper in the lower dosage group led to an increase in recurrence of symptoms that may have increased this apparent effect. The dosing recommendation from this study resulted in a set $4 \mathrm{mg} /$ day dosage with a dose taper for 28 days in patients with no symptoms of mass effect used for the second study.

Side effects appeared to be dose-dependent, occurring more frequently in patients using $16 \mathrm{mg} /$ day. Therefore, the authors concluded that steroid related toxicity was increased with the higher daily dosage. Although the study is double blind and randomized, the relatively small size, inconsistencies in the design and lack of clarity in the presentation of the data and analysis are concerns. Nonetheless, this work appears to be the most detailed and informative available to assist with formulating treatment recommendations. The data, while extremely limited, support a set of treatment recommendations which follow a general trend toward higher steroid dosages in symptomatic patients exhibiting greater mass effect symptoms of increased intracranial pressure, as opposed to lower doses of steroids for asymptomatic patients.

Wolfson et al. [6] prospectively studied 12 patients with histologically confirmed malignancies and radiographically documented brain metastases and attempted to evaluate the indications for glucocorticoids. Patients were scored for general performance status and neurologic function class. All subjects received $24 \mathrm{mg}$ of intravenous dexamethasone every $6 \mathrm{~h}$ for $48 \mathrm{~h}$ and after an assessment, were randomized to receive either $4 \mathrm{mg}$ of oral dexamethasone every $6 \mathrm{~h}$ (Group 1) or no steroids (Group 2) during radiotherapy (30 Gy in ten fractions). Prior to randomization, five (33\%) had a positive response and eight $(67 \%)$ had no response to the high dose level. Seven patients were randomized to continue receiving steroids at the reduced level and five received none during radiotherapy. The change in general performance status for Group 1 was: Improved 2/7 (29\%), deteriorated 1/7 (15\%), no change 4/7 (57\%). For Group 2, the change in performance status was: Improved $0 / 5(0 \%)$, deteriorated $1 / 5(20 \%)$ and no change $4 / 5(20 \%)$. The change in neurological function for Group 1 was: Improved $1 / 7(14 \%)$, deteriorated $1 / 7$ (14\%), no change 5/7 (72\%); change in neurological function for Group 2 was: Improved $0 / 5(0 \%)$, deteriorated $1 / 5(20 \%)$, no change $4 / 5(80 \%)$. No statistical analysis was performed due to the small sample size. The authors conclude that the specific role of steroids in treating patients with metastatic carcinoma to the brain remains uncertain and should be evaluated in a cooperative prospective trial. Due to the small size and lack of any statistical analysis, it is impossible to reach any conclusion based on this study; therefore, no recommendations will be made based on this small trial.

\section{Discussion}

Role of steroids in metastatic brain disease

Given the extremely limited number of studies which satisfied the conditions of our search criteria, an additional discussion of published literature on the subject of corticosteroids in metastatic brain disease is provided to offer a larger context for this topic. While the following studies were not part of the body of evidence considered in 
formulating treatment recommendations in our evidencebased guidelines, they do highlight areas of interest where clinical trials are still required to answer important steroidrelated questions.

A review of the outcomes in the Radiation Therapy Oncology Group (RTOG) studies examining various radiotherapeutic regimens for patients with metastatic brain disease demonstrated a dramatic early improvement in patients treated concurrently with steroids during the early phases of the trials [10]. However, as the authors note, the administration of steroids was not well controlled in these studies and was not a primary point of interest so, although these observations contributed to the overall clinical impression that steroids were of benefit in the adjuvant treatment of brain metastases, it is difficult to extrapolate treatment recommendations from these studies. In Borgelt et al., this is shown by the fact that symptomatic patients receiving steroids had a significant improvement in neurological function as opposed to those who did not receive steroids. None of these studies suggested that the use of steroids halted time to progression.

Gaspar et al. [7] published an American College of Radiology (ACR) Committee on Appropriateness Criteria consensus report following an expert panel review on the pre-irradiation evaluation and management of brain metastases. The authors cite six articles to support the generation of their expert opinions [5, 6, 10-13]. They initially note that although clinical experience has established the effectiveness of corticosteroids in reducing symptoms and radiographic peritumoral edema, controversy remains on the specific indications and dosage used. They point out that the earliest studies to support steroid use prior to radiotherapy used radiation dosing schemes that are presently atypical and that there was evidence that steroids were only indicated when there was a concern about raised intracranial pressure [10-12]. In one study, $27 \%$ of patients who received $10 \mathrm{~Gy}$ of single fraction whole brain radiotherapy (WBRT) experienced signs of increased intracranial pressure [12]. They also note evidence from one trial indicating that patients who were moderately symptomatic who received steroid therapy during radiation experienced a more rapid improvement in their clinical symptoms, although there was no impact on progression-free or overall survival. This review went onto outline several papers addressing the acute and chronic side effects and the studies that address dosing and toxicity $[5,6,13]$.

This consensus review summarizes the authors' views on steroid use for metastatic brain disease by indicating that the patient who shows evidence of elevated intracranial pressure, but who does not require immediate surgical attention for either hydrocephalus or impending herniation, should receive 4-6 mg/day of dexamethasone in divided doses and that the routine use of corticosteroids in patients without neurological symptoms is not necessary.

Effect of steroids on radiographic edema

In 1982, Hatam et al. [14] prospectively studied the effect of dexamethasone therapy on peritumoral edema based on computed tomography (CT) describing specifically three cases of metastatic intracranial disease in which a linear decrease in edema volume was observed, reducing the measured volume of edema to one-fourth of the initial volume after 2 weeks of treatment starting with $4 \mathrm{mg}$ four times daily and tapered to $1 \mathrm{mg} /$ day. The exact time course for the metastatic patients was not reported separately but the authors did report a substantial clinical improvement in these patients they felt was associated with the use of dexamethasone.

Andersen et al. [11] published a subsequent report in 1994 that quantified the effect of steroid therapy over time on peritumoral brain edema in vivo using a magnetic imaging based technique. Magnetic resonance based assessments of peritumoral edema were undertaken on 23 brain tumor patients including 13 with brain metastases at baseline and at Days 1, 3 and 7 after starting steroid therapy. Intravenous dexamethasone was used at dose of 0.26 $0.64 \mathrm{mg} / \mathrm{kg}$ (for a $70 \mathrm{~kg}$ patient this range is $20-45 \mathrm{mg}$ / day). After 7 days of steroid treatment the total edema area was reduced by $10.3 \%$ in the metastatic patients, with an average reduction in mean volume of $4.6 \%$ after $24 \mathrm{~h}$ of treatment and $13.5 \%$ after 7 days. This effect was observed for up to 63 days and it was demonstrated that after 40-63 days (6-9 weeks) of steroid therapy the peritumoral water content was close to the upper normal range for white matter. The authors concluded that steroids significantly reduce the volume of peritumoral edema in patients with metastatic brain disease even after a few days of treatment. They postulate a mechanism of steroid-induced reduction in edema below the level of absorption and commented that they thought they may have obtained the same effects with a dose-reduction to minimize potential side effects.

Minamikawa et al. [15] studied the effect of glucocorticoid treatment (methylprednisolone, dose not specified) on the apparent diffusion coefficient (ADC) imaging in a series of 13 patient including four with metastatic brain disease. Although it is not possible to examine the effects on the metastatic patients separately, the authors suggested that ADC imaging was a more sensitive technique than conventional MRI imaging for studying the effects of steroids on peritumoral edema, even in the absence of detectable MRI changes. 
Dosing and toxicity

Hempen et al. [13] retrospectively reviewed 138 consecutive patients to evaluate the impact of dosage and duration of dexamethasone administration during radiation therapy for patients with both primary and metastatic brain tumors $(n=91)$. The dosage of dexamethasone was gradually reduced from an initial median dose of 7-12 $\mathrm{mg} /$ day to a median of 1-6 mg/day with an average duration of 7 weeks for the metastatic group. The authors reported a period of initial clinical improvement with relatively few side effects. Specifically, $33 \%$ of patients reported improvement in symptoms with steroid use prior to radiotherapy, $44 \%$ during radiotherapy and $11 \%$ following radiotherapy. However, as dexamethasone was continued, this trend reversed and less symptom relief was observed with increasing toxicity. Life-threatening complications were rare. Side effects attributed to steroid use included: hyperglycemia (47\%), peripheral edema (11\%), psychiatric disorder (10\%), candidiasis (7\%), Cushing's syndrome (4\%), muscular weakness (4\%) and pulmonary embolus (2\%). Of a cohort of 13 clinically asymptomatic patients who received no dexamethasone during radiotherapy, 12 (92\%) showed no sign of worsening neurological problems. The patient with worsened symptoms had brain stem involvement. The authors conclude that dexamethasone effectively minimizes neurological symptoms and radiation therapy related side effects in patients with both primary and secondary brain tumors. The toxicity of dexamethasone was noted to increase over time and therefore a patient-specific dosing pattern and taper was recommended. The authors suggested that a prospective study specifically addressing the relative balance of symptom relief versus toxicity would be of benefit and that there is little evidence to support the use of steroids in the asymptomatic patient.

In 1999, Lagerwaard et al. [8] published an extensive retrospective review of 1,292 patients with metastatic brain disease referred to a single institution to identify prognostic (risk) factors. The majority of patients were treated with WBRT $(\mathrm{n}=1,079,84 \%) ; 118(9 \%)$ were treated with steroids only and 95 (7\%) with surgery and radiotherapy. Data obtained included: age, sex, performance status, number and distribution of brain metastases, site of primary tumor, histology, interval between primary tumor and brain metastases, systemic tumor activity, serum lactate dehydrogenase, response to steroid treatment, and treatment modality. Dexamethasone was used in a dose range of 4$16 \mathrm{mg} /$ day (mean dosage $14.6 \mathrm{mg} /$ day), which was "tapered slowly and discontinued in the weeks following therapy" (specific details not stated).

Response to steroid therapy was judged retrospectively, from review of the medical records, as good (marked improvement), moderate (some improvement) or little (little or no improvement). Both univariate and multivariate analyses were performed. The overall median survival was 3.4 months, with 6 month, 1 , and 2 year survival percentages of 36,12 , and $4 \%$, respectively. Median survival was 1.3 months in patients treated with steroids only, 3.6 months in patients treated with radiotherapy, and 8.9 months in patients treated with surgical resection followed by radiotherapy (all comparisons $P<0.0001$ ).

Multivariate analysis confirmed the previously reported observation that the impact of treatment modality on survival in patients with brain metastases was the most significant single factor in predicting survival. However, response to steroid treatment, performance status, systemic tumor activity and serum lactate dehydrogenase levels were also all independent prognostic factors on survival, second only to treatment modality in level of significance. Site of primary tumor, age, and number of brain metastases were also identified as prognostic factors.

The authors concluded that the strongest prognostic (risk) factors were choice of treatment modality, response to steroids, performance status, and evidence of systemic disease and they proposed using these classifiers in identifying favorable and unfavorable subgroups of patients with brain metastases for future studies.

Millar et al. [9] attempted to assess the impact of steroid therapy in the management of brain metastases by undertaking a systematic review of published randomized trials on WBRT for multiple cerebral metastases. In this extensive review published in 2004, the authors identified 21 full manuscripts of published randomized controlled trials involving WBRT in the treatment of multiple brain metastases from 1971 to 2003 . They attempted to extract details on the use and type of steroid, timing of steroids relative to radiotherapy, response assessment and contribution of steroids to overall outcome. They reported that 18 of the 21 trials reviewed documented steroid use. Overall survival was an outcome assessed in all studies. Thirteen studies assessed neurological response and ten assessed a radiographic endpoint. Ten of the studies (48\%) suggested a positive effect of steroid use on the outcome assessed. Only one study —in the palliative setting—provided a fixed steroid dose.

The authors concluded that the reporting of steroid use in previously published randomized trials assessing treatment for patients with brain metastases is "non-uniform and not sufficiently detailed" making it difficult to assess the treatment effect. They call for a more standard approach to steroid dose in future studies or at a minimum better reporting of dosages used to better determine symptom response and durability of response. They also note that side effects of steroid use and the ability to taper off steroids after treatment intervention are additional outcomes of interest in the planning of future studies. 
Weissman et al. [16] published their experience with a treatment regimen designed to reduce steroid toxicity in 20 patients with newly diagnosed brain metastases. All patients received dexamethasone starting at $8 \mathrm{mg}$ twice daily for 4 days then $4 \mathrm{mg}$ twice daily for 4 days followed by $2 \mathrm{mg}$ twice daily until the last day of radiation therapy. Radiation dose and schedules ranged from $20 \mathrm{~Gy}$ in five fractions to $58 \mathrm{~Gy}$ in 29 fractions. Fourteen patients (70\%) received dexamethasone for a minimum of $24 \mathrm{~h}$ before their first radiation treatment and seven of those patients (50\%) experienced improvement in neurologic symptoms and signs before radiotherapy. Fourteen patients $(70 \%)$ completed the entire course of radiation and dexamethasone as planned. One patient required reinstitution of dexamethasone within 30 days of finishing radiation for neurological decline. The authors felt that the twice-daily dose was well tolerated. Steroid related toxicity included hyperglycemia (5\%), candida esophagitis (5\%), steroid pseudorheumatism (10\%), peripheral edema (5\%) and steroid withdrawal syndrome $(5 \%)$.

In 2006, the results of a series of systematic reviews with the stated purpose "to establish evidence-based guidelines and identify controversies regarding the management of patients with brain metastases" was published by Soffietti et al. [1]. This was conducted by a multi-disciplinary task force of the European Federation of Neurological Societies and includes a review of data obtained from the Cochrane Library, bibliographic databases, overview papers and previous guidelines from scientific societies and organizations. Under the section on supportive care and steroids only one original article is cited and reviewed, and that is the publication by Vecht [5], which also met the eligibility criteria for this guideline paper and is discussed extensively in the scientific foundation section. This paper was used to support the recommendation that, in most cases, initial dexamethasone doses should not exceed 4-8 mg/day. However, in patients with more severe symptoms related to increased intracranial pressure doses of $16 \mathrm{mg} /$ day or higher should be considered (Level of Evidence B) [17]. The remainder of the recommendations were based on uniform consensus of the task force (referred to as a "Good Practice Point") and are summarized below. Dexamethasone was noted as the corticosteroid of choice and twice daily dosing was thought to be sufficient (Good Practice Point). Tapering of steroid dosing within 1 week of starting therapy and discontinuation within 2 weeks if possible was encouraged (Good Practice Point). Finally, patients who do not have signs or symptoms of increased intracranial pressure do not have to be treated with steroids (Good Practice Point).

Given the very limited number of studies that met the inclusion criteria for the systematic literature review on the role of steroids in metastatic brain disease, we are unable to propose specific guidelines on many of the issues of central interest to the treating physician.

In summarizing the Discussion section above we are able to offer the following observations: first, Overall the literature favors the position that corticosteroids are of benefit in providing temporary symptomatic relief of CNS symptoms related to increased intracranial pressure and edema secondary to brain metastases [1, 7-10, 13]. Secondly, dexamethasone is the steroid most often used, with the rationale that it has limited mineralocorticoid effects. Other steroids have been used with similar reported clinical responses. Dosing has been recommended to be sufficient at twice daily although more frequent dosing has been suggested with increasing concern for raised intracranial pressure and impending herniation $[1,7,8,11,14]$. Finally, based primarily on the asymptomatic patients in the study by Hempen et al., it is the opinion offered in the available studies that asymptomatic patients do not require steroids $[1,7,13]$. Numerous authors cite concerns for the toxicity of steroid therapy and due to the lack of a uniform recommendation on dosing and the apparent complexity of the response in individual patients it has been recommended that dose reduction be considered within 1 week of initiation of treatment. The dose reduction appears to often require alteration in individual patients due to the diversity of response [1, 7, 9, 13, 16].

An interesting observation in the review of the literature on this subject is the number of authors who appear to assume that steroids are a mainstay of treatment for the patient with metastatic brain disease despite the relative lack of detailed information available to guide specific therapy. In general, the evidence for specific dosages and regimens of steroids are poorly detailed. On the other hand, there is very little information provided in the literature that suggests that steroids are of no benefit in this patient population with the exception of the asymptomatic patient.

Given the general consensus in the literature that steroids are of benefit in selected patients with metastatic brain disease and the frequent observation that dosing needs to be specifically tailored to the individual, it appears unlikely that in depth studies specifically addressing this issue will be forthcoming. However, the need for additional recommendations for dosing and duration of therapy may result in an increased awareness of this concern and potentially an alteration of clinical trials designed to address comparisons.

\section{Summary and conclusions}

In terms of articles meeting the search criteria described in the methods section above, the study by Vecht et al. [5] 
stands alone in providing the most convincing data on the role for steroids in patients with brain metastases and for the choice of dosing. Based on their observations of improvement in all groups treated with steroids, a level 3 recommendation can be made as follows:

Steroid therapy versus no therapy

Corticosteroids are recommended to provide temporary symptomatic relief of CNS symptoms related to increased intracranial pressure and edema secondary to brain metastases. (Level 3 recommendation). Dexamethasone is the corticosteroid of choice, mainly because of its limited mineralocorticoid effects, and should be tapered slowly over several weeks to avoid rebound symptoms.

The Vecht et al. [5] article provides evidence that the administration of steroids provides relief of symptoms in patients with symptomatic brain metastatic disease; however, recognizing that there is no control group (a "no treatment group") only the lowest grade of recommendation can be made.

Vecht et al. [5] also conclude that a starting dose of 4-8 $\mathrm{mg} /$ day be considered, unless patients exhibit severe symptoms consistent with increased intracranial pressure. They further recommended that those patients at high risk for severe consequences from raised intracranial pressure be considered for more aggressive dosing on the order of doses of $16 \mathrm{mg} / \mathrm{day}$ or higher. This study is relied upon heavily in the systematic review and consensus reports summarized in the "Discussion" section above [1, 7, 9]. However, the number of patients involved is relatively small and the study lacks a validation group; thus, despite the randomized design and ranking yielding a higher class of evidence, the recommendation itself is downgraded to reflect these concerns. Based on this study design and results, the following recommendation can be made.

\section{Comparison of different doses of steroid therapy}

It is recommended for patients who are symptomatic from metastatic brain disease that a starting dose of $4-8 \mathrm{mg} / \mathrm{day}$ of dexamethasone be considered, unless patients exhibit severe symptoms consistent with increased intracranial pressure. In these patients it is recommended that higher doses such as $16 \mathrm{mg} /$ day or more be considered. (Level 3 recommendation)

\section{Key issues for further investigation}

Given the number of patients who receive steroids as a portion of their care for treating the signs and symptoms of brain metastases, the medical literature contains relatively few detailed reports specifically addressing this issue. It may be that the early observation that there was little effect on overall survival has limited the subsequent level of interest in this issue. Review of the literature finds that the majority of authors on the subject feel that there is a symptomatic benefit from the use of systemic steroids in the management of these patients. It is not clear that there is an urgent need for randomized trials specifically addressing the issue of steroid dosing and toxicity. However, future studies could be planned to allow better control, recording and analysis of steroid dosing and response to allow a more robust analysis of the risk to benefit ratio of various dosing regimens. This would potentially optimize the benefits while limiting the side effects, which could lead to an improvement in overall outcome in addition to improving quality of life measures in the short term.

\section{No ongoing or recently closed clinical trials on the use of steroids for the management of brain metas- tases were found that met the eligibility criteria.}

Acknowledgments We would like to acknowledge the contributions of the McMaster Evidence-based Practice Center (EPC), Dr. Parminder Raina, (Director). Dr. Lina Santaguida (Co-Associate Director, Senior Scientist) led the EPC staff, which was responsible for managing the systematic review process, searching for and retrieving, reviewing, data abstraction of all articles, preparation of the tables and the formatting and editing of the final manuscripts.

Disclaimer of liability The information in these guidelines reflects the current state of knowledge at the time of completion. The presentations are designed to provide an accurate review of the subject matter covered. These guidelines are disseminated with the understanding that the recommendations by the authors and consultants who have collaborated in their development are not meant to replace the individualized care and treatment advice from a patient's physician(s). If medical advice or assistance is required, the services of a competent physician should be sought. The proposals contained in these guidelines may not be suitable for use in all circumstances. The choice to implement any particular recommendation contained in these guidelines must be made by a managing physician in light of the situation in each particular patient and on the basis of existing resources.

Disclosures All panel members provided full disclosure of conflicts of interest, if any, prior to establishing the recommendations contained within these guidelines.

Open Access This article is distributed under the terms of the Creative Commons Attribution Noncommercial License which permits any noncommercial use, distribution, and reproduction in any medium, provided the original author(s) and source are credited.

\section{Reference}

1. Soffietti R, Cornu P, Delattre JY, Grant R, Graus F, Grisold W et al (2006) EFNS guidelines on diagnosis and treatment of brain metastases: report of an EFNS task force. Eur J Neurol 13(7): 674-681 
2. Robinson PD, Kalkanis SN, Linskey ME, Santaguida PL (2009) Methodology used to develop the AANS/CNS management of brain metastases evidence-based clinical practice parameter guidelines. J Neurooncol. doi:10.1007/s11060-009-0059-2

3. Centre for Evidence-Based Physiotherapy (2009) Physiotherapy evidence database (PEDro). http://www.pedro.org.au/. Accessed Jan 2009

4. Maher CG, Sherrington C, Herbert RD, Moseley AM, Elkins M (2003) Reliability of the PEDro scale for rating quality of randomized controlled trials. Phys Ther 83(8):713-721

5. Vecht CJ, Hovestadt A, Verbiest HB, van Vliet JJ, van Putten WL (1994) Dose-effect relationship of dexamethasone on Karnofsky performance in metastatic brain tumors: a randomized study of doses of 4, 8, and $16 \mathrm{mg}$ per day. Neurology 44(4): 675-680

6. Wolfson AH, Snodgrass SM, Schwade JG, Markoe AM, Landy H, Feun LG et al (1994) The role of steroids in the management of metastatic carcinoma to the brain. A pilot prospective trial. Am J Clin Oncol 17(3):234-238

7. Gaspar LE, Gutin PH, Rogers L, Schneider JF, Larson D, Bloomer WD et al (2000) Pre-irradiation evaluation and management of brain metastases. American College of Radiology. ACR appropriateness criteria. Radiology 215(Suppl):1105-1110

8. Lagerwaard FJ, Levendag PC, Nowak PJ, Eijkenboom WM, Hanssens PE, Schmitz PI (1999) Identification of prognostic factors in patients with brain metastases: a review of 1292 patients. Int J Radiat Oncol Biol Phys 43(4):795-803

9. Millar BM, Bezjak A, Tsao M, Sturdza A, Laperriere N (2004) Defining the impact and contribution of steroids in patients receiving whole-brain irradiation for cerebral metastases. Clin Oncol (R Coll Radiol) 16(5):339-344
10. Borgelt B, Gelber R, Kramer S, Brady LW, Chang CH, Davis LW et al (1980) The palliation of brain metastases: final results of the first two studies by the radiation therapy oncology group. Int $\mathrm{J}$ Radiat Oncol Biol Phys 6(1):1-9

11. Andersen C, Astrup J, Gyldensted C (1994) Quantitation of peritumoural oedema and the effect of steroids using NMRrelaxation time imaging and blood-brain barrier analysis. Acta Neurochir Suppl (Wein) 60:413-415

12. Harwood AR, Simson WJ (1977) Radiation therapy of cerebral metastases: a randomized prospective clinical trial. Int J Radiat Oncol Biol Phys 2(11-12):1091-1094

13. Hempen C, Weiss E, Hess CF (2002) Dexamethasone treatment in patients with brain metastases and primary brain tumors: do the benefits outweigh the side-effects? Support Care Cancer 10(4): 322-328

14. Hatam A, Yu ZY, Bergstrom M, Bergren BM, Greitz T (1982) Effect of dexamethasone treatment on peritumoral brain edema: evaluation by computed tomography. J Comput Assist Tomogr 6(3):586-592

15. Minamikawa $\mathrm{S}$, Kono $\mathrm{K}$, Nakayama $\mathrm{K}$, Yokote $\mathrm{H}$, Tashiro $\mathrm{T}$, Nishio A et al (2004) Glucocorticoid treatment of brain tumor patients: changes of apparent diffusion coefficient values measured by MR diffusion imaging. Neuroradiology 46(10):805-811

16. Weissman DE, Janjan NA, Erickson B, Wilson FJ, Greenberg M, Ritch PS et al (1991) Twice-daily tapering dexamethasone treatment during cranial radiation for newly diagnosed brain metastases. J Neurooncol 11(3):235-239

17. Brainin M, Barnes M, Baron JC, Gilhus NE, Hughes R, Selmaj K et al (2004) Guidance for the preparation of neurological management guidelines by EFNS scientific task forces-revised recommendations. Eur J Neurol 11:577-581 